\title{
BED SORE BIOMECHANICS
}




\section{BED SORE}

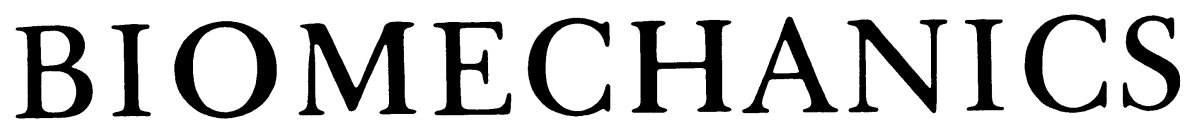

Proceedings of a seminar on Tissue Viability and Clinical Applications organised in association with the Department of Biomedical Engineering, the Institute of Orthopaedics (University of London), Royal National Orthopaedic Hospital, Stanmore, London, and held at the University of Strathclyde, Glasgow, in August, I975

Edited by

R. M. KENEDI and J. M. COWDEN

Bioengineering Unit

Wolfson Centre

University of Strathclyde

in association with

J. T. SCALES

Department of Biomedical Engineering

Royal National Orthopaedic Hospital

Stanmore

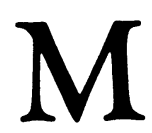


ISBN 978-1-349-02494-o ISBN 978-1-349-02492-6 (eBook) DOI 10.1007/978-1-349-02492-6

(C) Bioengineering Unit, University of Strathclyde 1976

Softcover reprint of the hardcover 1st edition 1976 978-0-333-17865-2

All rights reserved. No part of this publication may be reproduced or transmitted, in any form or by any means, without permission.

This book is sold subject to the standard conditions of the Net Book Agreement.

First published 1976 by

THE MACMILLAN PRESS LTD.

London and Basingstoke

Associated companies in New York, Dublin,

Melbourne, Johannesburg and Madras

SBN 333178653 


\section{PREFACE}

Bioengineering activities at the University of Strathclyde were initiated by the formation of a Medical Research Council Biomechanics Group in 1962. This has grown into the Bioengineering Unit, a research and postgraduate teaching department of the University housed in the Wolfson Centre.

The success of broad-spectrum symposia held in 1964 and 1972 has led to the establishment of the 'Strathclyde Bioengineering Seminars', an annual series the proceedings of which will be published by Macmillan Press. Each Seminar will explore in depth a clinically significant problem area within the Unit's activities.

The series was opened in August 1975 with a Seminar on the 'Biomechanics of Tissue Viability and Clinical Application to the Prevention of the Pressure Sore'. This particular Seminar was organised in association with the Department of Biomedical Engineering, Institute of Orthopaedics (University of London), Royal National Orthopaedic Hospital, Stanmore, London. Participation was gratifying: 207 attended, consisting of representatives from Australia, Canada, Denmark, England, France, India, Iraq, Israel, the Netherlands, Scotland, Sweden, the United States and Wales. In this context the organisers gratefully acknowledge the contribution of the National Fund for Research into Crippling Diseases towards travelling expenses of invited lecturers.

The details of the Scientific, Technical and Working Group Sessions are given in the contents list. There was also an Exhibition of Related Medical Engineering Equipment in which 19 firms and organisations participated (see list on page $x x i$ ). A visit to the Wolfson Centre was part of the programme, during which a variety of displays showing research projects in progress in the Bioengineering Unit were on show.

All the papers presented, together with additional contributions and the discussions, are printed complete in this volume, grouped in the six sections shown in the list of contents.

The organisers acknowledge with gratitude their sense of indebtedness to the Greater Glasgow Area Health Board for their support of the Seminar and for the very enjoyable open-air reception at the Wolfson Centre given by them in association with the University of Strathclyde, for participants and guests. They also wish to record their grateful thanks to the Institute of Orthopaedics, University of London, and to the Biological Engineering Society of the United 
Kingdom for their support and co-operation in assembling the programme and publicising the Symposium.

All the work connected with the Symposium as regards advance information, registration, accommodation arrangements, correspondence and the day-today running of the meetings was carried out by the staff of the Bioengineering Unit with the unstinted co-operation of the University Administration.

The Directors of the Armour Pharmaceutical Co. Ltd very kindly provided the pre-Seminar Dinner Reception, and by courtesy of the Bank of Scotland and Messrs. Arbuckle Smith \& Co., banking and travel agent facilities were made available to participants at the Seminar.

The organisers gratefully acknowledge the co-operation and whole-hearted assistance of all concerned.

R. M. KENEDI

University of Strathclyde, Glasgow, Scotland August 1975 
SEMINAR ORGANISING COMMITTEE AND SECRETARIAT

Joseph C. Barbenel

Frank Bell

Jane Bowen

James M. Courtney

Jennifer M. Cowden

Rita F. Cuthbert

Ronald Donovan

John H. Evans

Anne Johnston
Monica M. Jordan

Robert M. Kenedi (Chairman)

Flora Kennedy

Anne Lawson

James MacGregor

Sheila Milne

John P. Paul

John T. Scales

Rosemary Wilkinson

\section{SESSION REPORTERS}

Joseph C. Barbenel

Geoffrey I. Bardsley

Necip Berme

Elizabeth Christian

James M. Courtney

John H. Evans

Martin W. Ferguson-Pell

Maureen Gallagher

Keith Gilroy
Derek Jones

Peter Lawes

Arthur J. Loughran

David McClure

James MacGregor

Philip F. Millington

Paul Record

Peter J. Sharrock

Carolyn F. Small 


\section{LIST OF PARTICIPANTS AND AUTHOR INDEX}

(a, participating author; $a^{*}$, non-attending author; $c$, chairman and chairmanassociate)

ADAMSON, M. R., University of Strathclyde, Bioengineering Unit, Wolfson Centre, 106 Rottenrow, Glasgow $\mathrm{G}_{4}$ oNW, UK

ADAWAY, D., Sales Manager, Mycalex Instruments Ltd, Charlton Kings Industrial Estate, Cirencester Rd, Cheltenham GL53 8DZ, UK

AFFARA, Miss F., School of Nursing, Victoria Infirmary, Langside, Glasgow $\mathrm{G}_{4} 2 \mathrm{9TY}, \mathrm{UK}$

$a^{*}$ (page 32 7) ALBISSER, A. M., Ph.D., P.Eng., Department of Medical Engineering, Hospital for Sick Children, 555 University Avenue, Toronto, Ontario $\mathrm{M}_{5} \mathrm{G}_{1} \mathrm{X} 8$, Canada

$a$ (page 109) ALEXANDER, H., Ph.D., Associate Professor, Department of Mechanical Engineering, Stevens Institute of Technology, Castle Point Station, Hoboken, New Jersey o 7080, USA

AL-HUSSAINI, Professor J., Department of Physics, College of Science, University of Baghdad, Baghdad, Iraq

$a^{\bullet}$ (page 207) ANANTHAN, P. R., Division of Biomedical Engineering, Indian Institute of Technology, Madras 600036, India

$c, a$ (pages ANDERSON, Sir Ferguson, O.B.E., K.St.J., M.D., F.R.C.P. 127,181) \& S., Department of Geriatric Medicine, Stobhill Hospital, Glasgow $\mathrm{G}_{2} 13 \mathrm{UW}$, UK

ANDREWS, Dr J., Geriatric Unit, West Middlesex Hospital, Isleworth, Middlesex, UK

$a^{*}$ (page 207) ANTIA, N. H., The Tata Department of Plastic Surgery, Sir J. J. Group of Hospitals, Bombay 8, India

$a$ (pages 25, BARBENEL, J. C., B.D.S., M.Sc., L.D.S.(Eng.), University of 83, 225, Strathclyde, Bioengineering Unit, Wolfson Centre, 106 $344)$ $a$ (page 225) Rottenrow, Glasgow $\mathrm{G}_{4}$ oNW, UK

BARDSLEY, G. I., B.Sc., University of Strathclyde, Bioengineering Unit, Wolfson Centre, 106 Rottenrow, Glasgow $\mathrm{G}_{4}$ oNW, UK

BARR, Dr P-O, Burns Unit, Karolinska Hospital, 10401, Stockholm, Sweden 
$c, a$ (pages 55, BARTON, A. A., B.Sc., M.D., Department of Geriatric 257) Medicine, Nunnery Fields Hospital, Canterbury, Kent, UK BAXTER, Miss B., 596 Oldfield Lane, Greenford, Middlesex, UK

BEHAN, J. T., 173 Scott Street, Galashiels, Selkirkshire, UK

$a$ (pages 189 , BELL, F., B.Sc., Ph.D., C.Eng., M.I.Mech.E., University of 225) Strathclyde, Bioengineering Unit, Wolfson Centre, 106 Rottenrow, Glasgow $\mathrm{G}_{4}$ oNW, UK

BELL, Miss J. M., Principal Nursing Officer, General Nursing Division, Southern General Hospital, 1345 Govan Road, Glasgow $\mathrm{G}_{51}{ }_{4} \mathrm{TF}$, UK

BENDALL, T. J., Technical Manager, Hugh Steeper Ltd, Westland Square Industrial Estate, Westland Road, Leeds LS 11 ${ }_{4} \mathrm{SS}, \mathrm{UK}$

BENSON, G. B., G. D. Searle \& Co. Ltd, P.O. Box 11, Coronation Road, High Wycombe, Bucks, UK

$a$ (page 344) BERME, Dr N., University of Strathclyde, Bioengineering Unit, Wolfson Centre, 106 Rottenrow, Glasgow $\mathrm{G}_{4}$ oNW, UK

BISSETT, Miss E. M., Senior Nursing Officer, Langside Geriatric Unit (South Eastern District), Queens Park House, Langside Road, Glasgow $\mathrm{G}_{4} 2$ 9TT, UK

$a$ (page 235) BLACK, R., B.Sc., B.A.Sc., P.Eng., Rehabilitation Engineering, Ontario Crippled Children's Centre, 350 Rumsey Road, Toronto, Ontario $\mathrm{M}_{4} \mathrm{G}_{1} \mathrm{R} 8$, Canada

BOTS, R. A. A., Groesbeekseweg 362 , Nijmegen, the Netherlands

BOWEN, Miss J., University of Strathclyde, Bioengineering Unit, Wolfson Centre, 106 Rottenrow, Glasgow $\mathrm{G}_{4}$ oNW, UK

BOWKER, Dr P., University of Aberdeen, Department of Medical Physics, Foresterhill, Aberdeen AB 9 2ZD, UK

BOWMAN, Mrs N. K., Senior Nursing Officer, Philipshill Hospital, Busby, Clarkston, Glasgow, UK

$a *$ (page 103) BRAKKEE, A. J. M., Ph.D., Laboratorium voor Medische Fysica, Geert Grooteplein Noord 21 , Nijmegen, the Netherlands $c, a$ (pages 19, BRAND, Dr P. W., C.B.E., F.R.C.S., Chief, Rehabilitation 37,183 ) Branch, Department of Health, Education and Welfare, Public Health Service, US Public Health Service Hospital, Carville LA 70721 , USA

$c, a$ (pages, BRÅNEMARK, Professor P-I, Director of Research, University 63, 257) of Göteborg Faculty of Medicine, Department of Anatomy, Fack S-400-33, Göteborg, Sweden.

$a *$ (page 185) BRATTGÅRD, S. O., Department of Handicap Research, University of Göteborg, Ovre Husargatan 36, $S_{41314}$, Göteborg, Sweden 
BURKE, Dr D. C., Spinal Injuries Unit, Austin Hospital, Heidelberg, Victoria 3084, Australia

CAMPBELL, Miss M., Philipshill Hospital, Busby, Clarkston, Glasgow, UK

$a *$ (page 185) CARLSÖÖ, S., Department of Handicap Research, University of Göteborg, Ovre Husargatan 36, S 41314 , Göteborg, Sweden CAVINUE, Mrs M., 20 Peacock Loan, Carluke, ML8 5 RU, UK CHETTY, K. M., Remedial Gymnast, Spinal Unit, Philipshill Hospital, Busby, Clarkston, Glasgow, UK

$a *$ (page 69) CHIMOSKEY, J. E., M.D., University of Washington, Seattle, Washington 98195 , USA

$a^{*}$ (page 95) CHOW, W. M., Ph.D., Kenner Products, 912 Sycamore Street, Cincinnati, Ohio 45202 , USA

CHRISTIAN, Miss E., University of Strathclyde, Bioengineering Unit, Wolfson Centre, 106 Rottenrow, Glasgow $\mathrm{G}_{4}$ oNW, UK

$a$ (page 95) COCKRELL, J. L., Ph.D., Department of Physical Medicine and Rehabilitation, University of Michigan Medical Centre, Ann Arbor, Michigan 48104 , USA

CONDIE, D., Dundee Limb Fitting Centre, 133 Queen Street, Broughty Ferry, Dundee DD 5 1 AG, UK

$a^{*}$ (page 109) COOK, T. H., Ph.D., Stevens Institute of Technology, Hoboken, New Jersey o 7030, USA

COPELAND, K., General Secretary, Biological Engineering Society, Department of Biophysics, University of London, Gower Street, London WC1, UK

COURTNEY, Dr. J. M., University of Strathclyde, Bioengineering Unit, Wolfson Centre, 106 Rottenrow, Glasgow $\mathrm{G}_{4}$ oNW, UK

COWDEN, Miss J. M., University of Strathclyde, Bioengineering Unit, Wolfson Centre, 106 Rottenrow, Glasgow $\mathrm{G}_{4}$ oNW, UK

CRAIG, Miss E., Group Head of Occupational Therapy, Philipshill Hospital, Busby, Clarkston, Glasgow, UK

CROSS, J., 'Gaidrew', 33 Callander Drive, Larbet, Stirlingshire, UK

$a$ (page 39) CRUICKSHANK, C. N. D., M.D., M.R.C.P., F.R.C.Path., Director, M.R.C. Unit on the Experimental Pathology of Skin, The Medical School, The University, Birmingham 15, UK CUTHBERT, Mrs R., University of Strathclyde, Bioengineering Unit, Wolfson Centre, 106 Rottenrow, Glasgow G4 oNW, UK

DALLEY, C., 6o Blackett Place, Newington, Edinburgh, UK $a$ (page 69) DALY, C. H., Ph.D., Associate Professor, Department of Mechanical Engineering, University of Washington, Seattle, Washington 98195 , USA 
DEGREMONT, O., Vice-President, Medical Division, Dosapro Milton Roy S.A., 3 Rue D'Edimbourg 75008, Paris, France DOBBIE, A. I., Department of Health and Social Security, Room 312, 286 Euston Road, London NW 1 3DN, UK DONALDSON, Miss R., Victoria Infirmary, Langside, Glasgow $\mathrm{G}_{4} 2$ 9TY, UK

DONOVAN, R., University of Strathclyde, National Centre for the Training and Education in Prosthetics and Orthotics, 119 Rottenrow, Glasgow $\mathrm{G}_{4}$ oNW, UK

$a$ (page 315) DORNAN, J., M.B., Ch.B., F.R.C.P.(C), Department of Rehabilitation Medicine, Toronto Western Hospital, 399 Bathurst Street, Toronto, Ontario, Canada

DRAYCON, P. R., Product Group Manager, Smith \& Nephew Ltd, Bessemer Road, Welwyn Garden City, Herts AL 7 1 HF, UK

$a$ (pages 25, EVANS, J. H., B.Eng., M.Sc., Ph.D., University of Strathclyde, 189, 344) Bioengineering Unit, Wolfson Centre, 106 Rottenrow, Glasgow $\mathrm{G}_{4}$ oNW, UK

EVANS, R. E., Talley Surgical Instruments Ltd, 47 Theobald Street, Borehamwood, Herts, UK

$a$ (page 133) EXTON-SMITH, Professor, A. N., M.A., M.D., F.R.C.P. St. Pancras Hospital, University College Hospitals, 4 St. Pancras Way, London NW1 oPE, UK

FAGE, C. B., Acting Superintendent, Physiotherapy Dept., Foresthall Hospital, Springburn, Glasgow, UK

FAIRLEY, Dr A., Geriatric Unit Office, North Nottingham Teaching District, Sherwood Hospital, Hucknall Road, Nottingham $\mathrm{NG}_{5}{ }_{1} \mathrm{PD}$, UK

FAIRLEY, Dr D., 64 Terregles Avenue, Glasgow G41, UK

FERGUSON, J., University of Strathclyde, Bioengineering Unit, Wolfson Centre, 106 Rottenrow, Glasgow $\mathrm{G}_{4}$ oNW, UK

$a$ (page 189) FERGUSON-PELL, M. W., B.Sc., University of Strathclyde, Bioengineering Unit, Wolfson Centre, 106 Rottenrow, Glasgow $\mathrm{G}_{4}$ oNW, UK

$a$ (page 315) FERNIE, G. R., B.Sc. Ph.D., C.Eng., M.I.Mech.Eng., CoDirector, Amputee Centre, Toronto Hospital, 82 Buttonwood Avenue, Weston, Ontario, Canada

GALLAGHER, Miss M., University of Strathclyde, Bioengineering Unit, Wolfson Centre, 106 Rottenrow, Glasgow $\mathrm{G}_{4}$ oNW, UK

GERMANS, Dr F. H., Institute of Medical Physics, TNO, Da Costakade 45, Utrecht, the Netherlands 
GERRARD, D., Johnson \& Johnson Ltd, Southampton Road, Cosham, Portsmouth, Hants, UK

$a^{*}$ (page 207) GHISTA, D. N., Ph.D., Head, Division of Biomedical Engineering, Indian Institute of Technology, Madras 600036, India

GIBBS, Dr J. R., Egerton Ltd, 2 Walters Yard, High Street, Bromley, Kent, UK

$a^{*}$ (page 32 7) GIBSON, D. A., M.D., F.R.C.S.(Eng \& C.), Hospital for Sick Children, 555 University Avenue, Toronto, Ontario $\mathrm{MG}_{5}$ 1X8, Canada

$a *$ (page 83) GIBSON, F., B.Sc., Department of Mechanical Engineering, Paisley College of Technology, Paisley, UK

$c, a$ (pages 25, GIBSON, Professor T., M.B.Ch.B., D.Sc., F.R.C.S.(Ed.)., 344) F.R.C.S.(Glasg.), Regional Director, Plastic Surgery Unit, Canniesburn Hospital, Glasgow G61 1QL, UK

GILROY, K., University of Strathclyde, Bioengineering Unit, Wolfson Centre, 106 Rottenrow, Glasgow $\mathrm{G}_{4}$ oNW, UK

GOUDIE, W. W., 46 Waverley Crescent, Greenfaulds, Cumbernauld, Glasgow, UK

GOURLEY, Miss M., Southern General Hospital, 1345 Govan Road, Glasgow $\mathrm{G}_{51}{ }_{4} \mathrm{TF}$, UK

GRAHAM, Miss D., Southern General Hospital, 1345 Govan Road, Glasgow $\mathrm{G}_{51} 4 \mathrm{TF}$, UK

GRANT, Mrs E., Southern General Hospital, 1345 Govan Road, Glasgow $\mathrm{G}_{51}{ }_{4} \mathrm{TF}$, UK

$a^{*}$ (page 169) GRANT, R. A., Ph.D., D.Sc., Ecotech Systems (UK) Ltd, Poole, Dorset, UK

$a$ (page 311 ) GRANT, W. Russell, M.A., M.R.C.S., L.R.C.P., D.Phys.Med., Director, Rheumatology and Rehabilitation, Royal Hampshire County Hospital, Winchester, Hants, UK

GRAZEBROOK, M. W., Sales Director, Watkins \& Watson Ltd, 14 High Street, Chislehurst, Kent, UK

GRIFFITHS, Miss S., General Hospital, Ayresome Green Lane, Middlesbrough, Cleveland $\mathrm{TS}_{5}{ }_{5} \mathrm{AZ}$, UK

GUIDOIN, Dr. R. G., University of Strathclyde, Bioengineering Unit, Wolfson Centre, 106 Rottenrow, Glasgow $\mathrm{G}_{4}$ oNW, UK GUNN, W. F., Scottish Home and Health Department, Pentland House, 47 Robb's Loan, Edinburgh EH 14 1TY, UK

$a$ (page 153) GUTTMANN, Sir Ludwig, C.B.E., M.D., F.R.C.P., F.R.C.S., Director, Stoke Mandeville Sports Stadium for the Paralysed and Other Disabled, British Paraplegic Sports Society Ltd, Harvey Road, Aylesbury, Bucks, UK

HARDY, Dr. A. G., Consultant. in Charge, Spinal Injuries Unit, 
Lodge Moor Hospital, Sheffield $\mathrm{S}_{10}$ 4LH, UK

$a$ (page 269) HARGEST, T. S., B. A., Director, Division of Clinical Engineering, Medical University of South Carolina, 8o Barre Street, Charleston, South Carolina 29401, U.S.A.

HENDERSON, Miss M., Philipshill Hospital, Busby, Clarkston, Glasgow, UK

HOLDSWORTH, L., Department of Health and Social Security, Kingston By-Pass Road, Surbiton, Surrey KT6 5 QN UK

$a^{\bullet}$ (page 69) HOLLOWAY, G. A., M.D., University of Washington, Seattle, Washington 98195 , USA

HORNE, Miss C., 35 Church Street, Alloa, Clackmannanshire, UK

HORRELL, P., Johnson \& Johnson Ltd, 160 Bath Avenue, Slough, Berks, UK

$c$ (page 344) HUGHES, J., B.Sc., C.Eng., M.I.Mech.E., F.I.S.P.O., University of Strathclyde, National Centre for Training \& Education in Prosthetics and Orthotics, 119 Rottenrow, Glasgow $\mathrm{G}_{4}$ oNW, UK

$a^{\bullet}$ (page 169) HULME, M. J., B.Sc., Department of Biological Sciences, University of Dundee, Dundee DD $14 \mathrm{HN}$, UK

HUTCHISON, Miss W., District Nursing Officer, Argyll and Clyde Health Board, Dumbarton District, Westonlee House, Bonhill Road, Dumbarton, UK

$a^{\bullet}$ (page 243) HUTTON, W. C., Polytechnic of Central London, 115 New Cavendish Street, London, W1M 8JS, UK

ILAHI, M., 845 Great West Road, Isleworth, Middlesex, UK

c(page 3) ILLINGWORTH, Sir Charles, C.B.E., M.D., Ch.M., D.Sc., LL.D., S.R.C.S., S.A.C.S., 57 Winton Drive, Glasgow G 12 , UK

$a$ (page 165) ISHERWOOD, P. A., M.B., Ch.B., Department of Health and Social Security, Biomedical Research and Development Unit, Roehampton Lane, London SW 15 PRR, UK

$a^{\bullet}$ (page 199) JACOBS, J. E., Ph.D., D.Sc., Director, Biomedical Engineering Centre, North Western University, Evanston, Illinois 60201, USA

$a$ (page 287) JENEID, Miss P., S.R.N., B.T.A., Regional Plastic and Jaw Surgery Centre, Burns Unit, Mount Vernon Hospital, Northwood, Middlesex, UK (Seconded from the Department of Biomedical Engineering, Institute of Orthopaedics, RNOH, Stanmore, Middlesex, UK)

JENKINS, Miss E., University of Strathclyde, Bioengineering Unit, Wolfson Centre, 106 Rottenrow, Glasgow $\mathrm{G}_{4}$ oNW, UK 
JOHNSTON, Miss A., University of Strathclyde, Bioengineering Unit, Wolfson Centre, 106 Rottenrow, Glasgow $\mathrm{G}_{4}$ oNW, UK JORDAN, Miss M., University of Strathclyde, Bioengineering Unit, Wolfson Centre, 106 Rottenrow, Glasgow $\mathrm{G}_{4}$ oNW, UK

$a$ (page 95) JUVINALL, R. C., M.S.M.E., Department of Mechanical Engineering, The University of Michigan, College of Engineering, Ann Arbor 48104, USA

$c$ (page 343) KENEDI, Professor R. M., B.Sc., Ph.D., A.R.C.S.T., C.Eng., F.I.Mech.E., M.R.Ae.S., F.R.S.E., Head, Bioengineering Unit, Wolfson Centre, University of Strathclyde, 106 Rottenrow, Glasgow $\mathrm{G}_{4}$ oNW, UK

$a *$ (page 69) KENNEDY, D., University of Washington, Seattle, Washington 98195 , USA

KENNEDY, Mrs F., University of Strathclyde, Administration Office, Royal College Building, George Street, Glasgow G 1 1XW, UK KIRBY, L., 12 Lower Road, Stoke Mandeville, Bucks, UK

$a^{*}$ (page 32 7) KORESKA, J., M.A.Sc., M.Ing.F., P.Eng., Department of Medical Engineering, Hospital for Sick Children, 555 University Avenue, Toronto, Ontario, M 5 G 1X8, Canada

LANKERSTER, M. J., Egerton Ltd, Walters Yard, High Street, Bromley, Kent, UK

LAWES, P., University of Strathclyde, Bioengineering Unit, Wolfson Centre, 106 Rottenrow, Glasgow $\mathrm{G}_{4}$ oNW, UK LAWSON, Mrs A., University of Strathclyde, Bioengineering Unit, Wolfson Centre, 106 Rottenrow, Glasgow $\mathrm{G}_{4}$ oNW, UK LAY, J., Medical Products Manager, Medelec Ltd, Manor Way, Old Woking, Surrey GU 22 9JD, UK

LEGGAT, Mrs M.A., Philipshill Hospital, Busby, Clarkston, Glasgow, UK

LILDHOLT, T., Teglgaardsvej 3, DK 8270, Hoejbjerg, Denmark

LONG, D. J., Technical Director, Mycalex Instruments Ltd, Charlton Kings Industrial Estate, Cirencester Road, Cheltenham GL53 8DZ, UK

LOUGHRAN, A., University of Strathclyde, Bioengineering Unit, Wolfson Centre, 106 Rottenrow, Glasgow $\mathrm{G}_{4}$ oNW, UK

$a$ (page 141) LOWTHIAN, P. T., S.R.N., Epidemiology and Medical Care Unit, Northwick Park Hospital, Watford Road, Harrow, Middlesex HA ${ }_{3}$ DJ, UK

LYON, H. F., Ellangowan, 18 Keir Street, Bridge of Allan, Stirling FK9 4 AY, UK 
LYTHE, Professor S. G. E., M.A., F.S.A.(Scot.), Vice Principal, University of Strathclyde, McCance Building, Rịchmond Street, Glasgow $\mathrm{G}_{1}$ 1XQ, UK

McCALL, Mrs J., District Nursing Officer, Southern General Hospital, Govan Road, Glasgow $\mathrm{G}_{51} 4_{\mathrm{TF}}$, UK MCCLURE, D., University of Strathclyde, Bioengineering Unit, Wolfson Centre, 106 Rottenrow, Glasgow, $\mathrm{G}_{4}$ oNW, UK McCONVille, Miss, C., 64 Campsie Road, Wishaw, Lanarkshire, UK

$a$ (page 147) McCREADIE, D.W.A., Ph.D., M.F.C.M., F.H.A., Scottish Home and Health Department, St. Andrews House, Edinburgh EH ${ }_{3}$ DT, UK

McDONALD, Miss V., Philipshill Hospital, Busby, Clarkston, Glasgow, UK

$a$ (page 161) McDOUGALL, A., M.B., Ch.B., F.R.C.S.(Edin.), F.R.C.S. (Glasg.), Department of Orthopaedics, Victoria Infirmary, Glasgow $\mathrm{G}_{42}$ 9TY, UK

MacGREGOR, Dr J., University of Strathclyde, Bioengineering Unit, Wolfson Centre, 106 Rottenrow, Glasgow $\mathrm{G}_{4}$ oNW, UK McGRouTHER, D. A., Plastic Surgery Unit, Canniesburn Hospital, Glasgow G61 1 QL, UK

MACINTYRE, Dr I. D., 327 North Christina, Sarnia, Canada MacKENZIE, Mrs E., Philipshill Hospital, Busby, Clarkston, Glasgow, UK

MACKIE, R., 32 Waverly Drive, Wishaw, Lanarkshire, UK MacLACHLAN, D. F. A., Gaeltec Ltd, Stein, Waternish, Isle of Skye, Invernesshire $\mathrm{IV}_{4}$ 19QA, UK

McMAHON, Miss M., Senior Nursing Officer, 3 Cumming Drive, Mount Florida, Glasgow $\mathrm{G}_{4} 2{ }_{9} \mathrm{~A}_{1}$, UK

McROBERTS, Mrs E., Southern General Hospital, 1345 Govan Road, Glasgow $\mathrm{G}_{51} 4^{\mathrm{TF}}$, UK

MARKS, Dr R., Consultant Physician, Department of Medicine, Welsh National School of Medicine, Heath Park, Cardiff $\mathrm{CF}_{4}$ $4 \mathrm{XN}, \mathrm{UK}$

MELROSE, Miss A. L., Knowepark Hospital, Galashiels, Selkirkshire, UK

MIDDLETON, B. A., Clinical Adhesives, 110 Leavesden Road, Watford, Herts, UK

MILLINGTON, Dr P. F., University of Strathclyde, Bioengineering Unit, Wolfson Centre, 106 Rottenrow, Glasgow $\mathrm{G}_{4}$ aNW, UK

MILNE, Miss S., University of Strathclyde, Bioengineering Unit, Wolfson Centre, 106 Rottenrow, Glasgow $\mathrm{G}_{4}$ oNW, UK 
MOLNAR, G., Armour Pharmaceutical Co. Ltd, Hampden Park, Eastbourne, Sussex BN 22 9AG, UK

MOORE, Miss M. J., Nursing Officer, 7 Banchory Avenue, Eastwood, Glasgow $\mathrm{G}_{43}$ 1 EX, UK

MORRISO N, Miss C., Victoria Infirmary, Grange Road, Glasgow $\mathrm{G}_{42}$ 9TY, UK

MORRISON, Miss J., General Hospital, Ayresome Green Lane, Middlesbrough, Cleveland $\mathrm{TS}_{5}{ }_{5} \mathrm{AZ}, \mathrm{UK}$

$a *$ (page 169) MUDIE, A., Department of Biological Sciences, University of Dundee, Dundee DD ${ }_{4} \mathrm{HN}, \mathrm{UK}$

$c$ (pages 125, MURDOCH, Professor G., M.B., Ch.B., F.R.C.S.(Edin.), 344)

Surgeon in Charge, Dundee Limb Fitting Centre, 133 Queen Street, Broughty Ferry, Dundee $\mathrm{DD}_{4} 1 \mathrm{AG}$, UK

MURRAY, Miss I., 88 Myrtle Crescent, Kirkcaldy, Fife, UK

NAIRN, Miss M., Chief Area Nursing Officer, Greater Glasgow Health Board, 351 Sauchiehall Street, Glasgow G 2 3HT, UK

NEALE, Mrs E., Philipshill Hospital, Busby, Clarkston, Glasgow, UK

NEUMARK, O. W., Beaufort (Air-Sea) Equipment Ltd, Wren Nest Mill, High Street West, Glossop, Derbyshire SK 13 8EZ, UK NICOLL, Miss E., Philipshill Hospital, Busby, Clarkston, Glasgow, UK

NUNN, Mrs G., Philipshill Hospital, Busby, Clarkston, Glasgow, UK

$a^{*}$ (page 169) OLIVER, R. F., B.Sc., Ph.D., Department of Biological Sciences, University of Dundee, Dundee DD $14 \mathrm{HN}, \mathrm{UK}$

PAGE, D. C., Dylade Co. Ltd, 45 Brindley Road, Astmoor Industrial Estate, Runcorn, Cheshire, UK

PARROT, C., Sales Manager, Dunlop Ltd, Dunlopillo Division, Pannal, Harrogate, North Yorks $\mathrm{HG}_{3}$ 1JL, UK

PATTERSON, J., University of Strathclyde, Bioengineering Unit, Wolfson Centre, 106 Rottenrow, Glasgow $\mathrm{G}_{4}$ oNW, UK

$c$ (page 3) PAUL, Professor J. P., University of Strathclyde, Bioengineering Unit, Wolfson Centre, 106 Rottenrow, Glasgow $\mathrm{G}_{4}$ oNW, UK

$a$ (page 219) PETERSEN, N. C., M.D., Surgeon in Charge, Department of Plastic Surgery, Århus University Hospital, 8000 Århus C., Denmark

PIGOTT, R. W., Consultant Plastic Surgeon, Frenchay Hospital, Bristol, UK

RECORD, P., University of Strathclyde, Bioengineering Unit, 
xviii

$a($ page 211$)$

Wolfson Centre, 106 Rottenrow, Glasgow $\mathrm{G}_{4}$ oNW, UK

REDFERN, Miss S. J., B.Sc., S.R.N., Applied Psychology

Department, University of Aston, College House, Costa Green,

Birmingham $\mathrm{B}_{4}{ }_{7} \mathrm{ET}$, UK

REHANA, Miss B., Doncaster Royal Infirmary, Doncaster, South Yorkshire, UK

REID, Miss A. A., Cameron Hospital, Windygates, Fife, UK

$c, a$ (pages REID, Miss E. A., M.Phil., R.G.N., R.S.C.N., S.C.M., Senior

147, 344) Nursing Adviser, Scottish Health Service, Common Services Agency, Buildings Division, 11 Drumsheugh Gardens, Edinburgh, UK

RENFREW, Mrs J., The Elms, 11 Grove Park, Lenzie, near Glasgow, UK

$c, a$ (pages RESWICK, J. B., Sc.D., Director, Rehabilitation Engineering 181 , 301 , Centre, Rancho Los Amigos Hospital, H121, 743 Golandrinas

344) Street, Downey, California 90242, USA

$a$ (page 5) ROAF, Professor R., F.R.C.S., M.Ch., Orth., M.A. (Oxon), Department of Orthopaedic Surgery, The University of Liverpool, P.O. Box 147, Liverpool L69 3BX, UK

ROBERTSON, J., University of Strathclyde, Bioengineering Unit, Wolfson Centre, 106 Rottenrow, Glasgow $\mathrm{G}_{4}$ oNW, UK

$a$ (page 301) ROGERS, J. E., M.S., 1400 North Carol Street, Lahabra, California, USA

$a$ (page 79) ROMANUS, E. Mikael, M.D., University of Göteborg, Faculty of Medicine, Department of Anatomy, Fack, S-400, 33, Göteborg, Sweden

ROPER, Miss N., M.Phil., S.R.N., R.S.C.N., R.N.T., Nursing Officer (Research), Room 25, St. Andrews House, Edinburgh EH 13 DE, UK

RYAN, D. M., 22 Viewfield Cottages, Stoney Chryston, near Glasgow, UK

SACHS, Dr. M. L., Hospital of the University of Pennsylvania, 3400 Spruce Street, G 1, Philadelphia 19104, USA

c, $a$ (pages 1 1, SCALES, Professor J. T., F.R.C.S., C.I.Mech.E., Department of 125,259 , Biomedical Engineering, The Institute of Orthopaedics, Royal 344) National Orthopaedic Hospital, Stanmore, Middlesex $\mathrm{HA}_{7}{ }_{4} \mathrm{LP}$, UK

SCOTT, I., Biomedical Engineering, 42/43 Gerrard Street, London $\mathrm{W}_{1} \mathrm{~V}{ }_{7} \mathrm{LP}, \mathrm{UK}$

SCOTT, Miss K. A., Nursing Officer, South Eastern District, Victoria Infirmary, Glasgow $\mathrm{G}_{42} 9 \mathrm{TY}$, UK

$a$ (page 185) SEVERINSSON, K., University of Göteborg, Department of Handicap Research, Ovre Husargatan 3, S 413-1, Göteborg, 
Sweden

SHARROCK, P. J., University of Strathclyde, Bioengineering Unit, Wolfson Centre, 106 Rottenrow, Glasgow $\mathrm{G}_{4}$ oNW, UK SHAVITT, Mrs C., 25 Rechov Hamelachim, Ramat, Hasharon, Israel

SIMMETT, I. F., University of Strathclyde, Bioengineering Unit, Wolfson Centre, 106 Rottenrow, Glasgow $\mathrm{G}_{4}$ oNW, UK

$c$ (page 37) SIMPSON, Professor D. C., M.B.E., B.Sc., Ph.D., F.R.S.E., Orthopaedic and Bioengineering Unit, Princess Margaret Rose Hospital, Edinburgh EH $107 \mathrm{ED}, \mathrm{UK}$

SKARRETT, R., Senior Nurse Tutor, 9 Ensford Close, Four Oaks, Sutton Coldfield, Midlands B 74 4LR, UK

SMALL, Miss C. F., University of Strathclyde, Bioengineering Unit, Wolfson Centre, 106 Rottenrow, Glasgow $\mathrm{G}_{4}$ oNW, UK

$a$ (page 321 ) SMITH, J. E., F.H.A., Director of Supply, Scottish Health Service, Common Services Agency, Pentland House, 47 Robbs Loan, Edinburgh EH 14 1TY, UK

SMITH, M., Physiotherapist, Spinal Unit, Philipshill Hospital, Busby, Clarkston, Glasgow, UK

SPEARS, Miss A., Scottish Home and Health Department, Pentland House, 47 Robbs Loan, Edinburgh, EH 14 1TY, UK SPEEDIE, Miss G., Department of Humanities, Glasgow College of Technology, North Hanover Place, Glasgow $\mathrm{G}_{4}$ oBA, UK $\mathrm{G}_{4}$ oBA, UK

SQUIRES, C., Area Manager, Smith \& Nephew Ltd, Bessemer Road, Welwyn Garden City, Herts $\mathrm{AL}_{7} \mathrm{1HF}$, UK

$a^{*}$ (page 207) SRINIVASAN, T. M., Division of Biomedical Engineering, Indian Institute of Technology, Madras 600036, India

STEPHENSON, A., Armour Pharmaceutical Co. Ltd, Hampden Park, Eastbourne, Sussex BN 22 9AG, UK

$a$ (page 2 77) STEWART, I. M., M.B., F.R.C.S.(Ed.), Orthopaedic Department, Law Hospital, Carluke, Lanarkshire ML8 ${ }_{4}$ ER, UK

$a^{*}$ (page 103) STIENEN, G. J. M., M.Sc.(Phys. Eng)., Laboratorium voor Medische Fysica, Geert Groeteplein Noord 21, Nijmegen, the Netherlands

$a$ (page 243) STOKES, I. A. F., Polytechnic of Central London, 115 New Cavendish Street, London W1M 8JS, UK

TAIT, J., Department of Health \& Social Security, Alexander Fleming House, Elephant and Castle, London SE 1 6BY, UK

TANNAHILL, Mrs M. B., Research Fellow, Department of Marketing, University of Strathclyde, Stenhouse Building, 143 Cathedral Street, Glasgow $\mathrm{G}_{4}$ oRQ, UK

$a$ (page 83) TURNBULL, F. W., B.Sc., University of Strathclyde, Bio- 
engineering Unit, Wolfson Centre, 106 Rottenrow, Glasgow $\mathrm{G}_{4}$ oNW, UK

TURNER, Dr N. A., 35 Colin Street, West Perth 6oo5, Australia

U'REN, E., Sales Manager, Talley Surgical Instruments Ltd, 47 Theobald Street, Borehamwood, Herts WD6 ${ }_{4}$ RT, UK

$a^{*}$ (page 103) VENDRICK. A. J. H., Ph.D., Laboratorium voor Medische Fysica, Geert Grooteplein Noord 21 , Nijmegen, the Netherlands

WALKER, Mrs C., School of Nursing, Victoria Infirmary, Langside, Glasgow $\mathrm{G}_{42}$ 9TY, UK

WATSON, D., Hugh Steeper Ltd, Westland Square Industrial Estate, Westland Road, Leeds $\mathrm{LS}_{11}{ }_{4} \mathrm{SS}$, UK

WHIMSTER, Dr I. W., Department of Dermatology, St. Thomas' Hospital, London SE $17 \mathrm{EH}$, UK

WIJKMANS, Dr D. W., Institute of Medical Physics TNO, Da Costakade 45, Utrecht, the Netherlands

$a$ (page 103) WIJN, P. F. F., M.Sc.(Phys. Eng.), Laboratorium voor Medische Fysica, Geert Grooteplein Noord 21 , Nijmegen, the Netherlands WILKINSON, Dr R., University of Strathclyde, Bioengineering Unit, Wolfson Centre, 106 Rottenrow, Glasgow $\mathrm{G}_{4}$ oNW, UK WILliAMSON, Mrs. J., General Hospital, Ayresome Green Lane, Middlesbrough, Cleveland $\mathrm{TS}_{5}{ }_{5} \mathrm{AZ}$, UK

$a$ (page 47) WINTER, G. D., B.Sc., Ph.D., Department of Biomedical Engineering, The Institute of Orthopaedics, Royal National Orthopaedic Hospital, Stanmore, Middlesex HA 74 LP, UK WOOD, Mrs M. A., Hillview, Inverbay, Invergowrie, Dundee $\mathrm{DD}_{2}$ 5 DG, UK 


\section{EXHIBITORS}

AGA Infrared Systems, Arden House, West Street, Leighton Buzzard, Beds LU 7 ${ }_{7} \mathrm{ND}$

Beaufort (Air-Sea) Equipment Ltd, Wren Nest Mill, High Street West, Glossop, Derbyshire SK 13 8EZ

Biological Engineering Society-General Secretary, K. Copeland, Esq., Department of Biophysics, University of London, London

Devices Limited, Instrument Division, Hyde Way, Welwyn Garden City, Herts $\mathrm{AL}_{7} 3 \mathrm{AP}$

Dunlop Ltd, Dunlopillo Division, Pannal, Harrogate, North Yorks $\mathrm{HG}_{3} 1 \mathrm{JL}$

Dylade Co. Ltd, 45 Brindley Road, Astmoor Industrial Estate, Runcorn, Chesire

Egerton Hospital Equipment Ltd, Tower Hill, Horsham, Sussex RH13 7JT Gaeltec Limited, Stein, Waternish, Isle of Skye, Inverness-shire IV 5 19QA

Henleys Medical Supplies Ltd, Alexandra Works, Clarendon Road, Hornsey, London N8 oDL

Instron Limited, Coronation Road, High Wycombe, Bucks

Johnson \& Johnson Limited, 260 Bath Road, Slough, Bucks SL $1{ }_{4}$ EA

Law Hospital, Department of Orthopaedics, Carluke, Lanarkshire ML8 5 ER

Medelec Limited, Manor Way, Old Woking, Surrey GU 22 9JU

Mycalex Instruments Ltd, Charlton Kings Industrial Estate, Cirencester Road, Cheltenham GL53 8DZ

Princess Margaret Rose Hospital, Bioengineering Unit, Fairmilehead, Edinburgh $\mathrm{EH}_{1} \mathrm{O}_{7} \mathrm{ED}$

John Smith \& Son (Glasgow) Ltd, Booksellers, 57 St. Vincent Street, Glasgow $\mathrm{G}_{2}$

Hugh Steeper Limited, Westland Square Industrial Estate, Westland Road, Leeds LS 115 SS

Talley Surgical Instruments Ltd, 47 Theobald Street, Borehamwood, Herts WD6 ${ }_{4} \mathrm{RT}$

Watkins \& Watson Ltd, Westminster Road Industrial Estate, Wareham, Dorset $\mathrm{BH}_{20} \mathrm{SP}$ 


\section{CONTENTS}

Preface v v

Seminar Organising Committee and Secretariat, and Session Reporters vii

List of Participants and Author Index ix

Exhibitors $\quad x x i$

Opening Remarks and Welcome to Participants 1

Professor S. G. E. Lythe, M.A., F.S.A. (Scot.)

\section{SESSION A PRESSURE SORES-THE PROBLEM}

The causation and prevention of bed sores $\quad 5$

R. Roaf

Pressure on the patient

J. T. Scales

Pressure sores-the problem $\quad 19$

P. W. Brand

Biomechanical concepts and effects

T. Gibson, J. C. Barbenel, J. H. Evans

Session A Discussion

\section{SESSION B TISSUE BIOMECHANICS}

The microanatomy of the epidermis in relation to trauma 39

C. N. D. Cruickshank

$\begin{array}{ll}\text { Some factors affecting skin and wound healing } & 47\end{array}$

G. D. Winter

The pathogenesis of skin wounds due to pressure $\quad 55$

A. A. Barton

$\begin{array}{ll}\text { Microvascular function at reduced flow rates } & 63\end{array}$

P-I Brånemark

The effect of pressure loading on the blood flow rate in human skin

C. H. Daly, J. E. Chimoskey, G. A. Holloway, D. Kennedy 
Microcirculatory reactions to controlled tissue ischaemia and temperature: a vital microscopic study on the hamster's cheek pouch

E. M. Romanus

Optical assessment of skin blood content and oxygenation

J. C. Barbenel, F. Gibson, F. Turnbull

Effects and characteristics of cushion covering membranes

W. W. Chow, R. C. Juvinall, J. L. Cockrell

Mechanical properties of the human skin in vivo for small deformations;

a comparison of uniaxial strain and torsion measurements

P. F. F. Wijn, A. J. M. Brakkee, G. J. M. Stienen, A. J. H. Vendrick

Variations with age in the mechanical properties of human skin in vivo

H. Alexander, T. Cook

Session B Discussion

SESSION C THE 'STATE OF THE ART'

IN PREVENTION, CURE AND COST EFFECTIVENESS

Preventive aspects of geriatric medicine

Sir Ferguson Anderson

Prevention of pressure sores: monitoring mobility and assessment of clinical condition

A. N. Exton-Smith

Underpads in the prevention of decubiti

P. T. Lowthian

Hospital accommodation for paraplegics

E. A. Reid, D. W. A. McCreadie

The prevention and treatment of pressure sores

Sir Ludwig Guttmann

Clinical aspects of bed sore prevention and treatment

A. McDougall

The use of chemically dried air in the treatment of chronic ulcers

P. A. Isherwood

The incorporation of trypsin-purified dermal collagen allografts into cutaneous wounds in the rat

R. F. Oliver, R. A. Grant, M. J. Hulme, A. Mudie

Session C Discussion

\section{SESSION D PATIENT MONITORING}

Patient monitoring

P. W. Brand 
Temperature and humidity in the sitting area

S. O. Brattgård, S. Carlsöö, K. Severinsson

Interface pressure sensors: existing devices, their suitability and limitations

M. W. Ferguson-Pell, F. Bell, J. H. Evans

A new multi-channel non-invasive pulse profile recording system

J. E. Jacobs

A pressure warning system for patients with insensitive feet to avoid ulceration

P. R. Ananthan, T. M. Srinivasan, N. H. Antia, D. N. Ghista

The comfort of the hospital bed

S. J. Redfern

The development of pressure sores during hospitalisation

N. C. Petersen

A technique for monitoring body movements during sleep

G. I. Bardsley, F. Bell, J. C. Barbenel

Suspension seating

R. Black

The effect of the diabetic ulcer on the load-bearing function of the foot

I. A. F. Stokes, W. C. Hutton

Session D Discussion

\section{SESSION E BODY SUPPORT CHARACTERISTICS}

Air support systems for the prevention of bed sores

259

J. T. Scales

Problems of patient support: the air fluidised bed as a solution

T. S. Hargest

Sand bed nursing

I. M. Stewart

Static and dynamic support systems-pressure differences on the body

P. Jeneid

Experience at Rancho los Amigos Hospital with devices and techniques to prevent pressure sores

J. B. Reswick, J. Rogers

Mobile and static support systems

W. Russell Grant

The problems of clinical trials with new systems for preventing or healing decubiti

G. R. Fernie, J. Dornan

Economic aspects of unconventional beds and support surfaces

J. E. Smith 
$x x v i$ CONTENTS

A novel support system for unstable spines

J. Koreska, A. M. Albisser, D. A. Gibson

\section{SESSION F REPORT OF WORKING GROUPS}

Group 1. Clinical aspects of prevention, therapy and cost effectiveness

Group 2. Identification of controlling parameters

Group 3. Patient management with specific reference to nursing techniques

Index 